Cinémas

Revue d'études cinématographiques

Journal of Film Studies

\title{
Romance du livre et du film : l'adaptation de la Romance du livre et de l'épée par Ann Hui
}

\section{Stephanie Hoare}

Volume 3, numéro 2-3, printemps 1993

Le nouveau cinéma chinois

URI : https://id.erudit.org/iderudit/1001196ar

DOI : https://doi.org/10.7202/1001196ar

Aller au sommaire du numéro

Éditeur(s)

Cinémas

ISSN

1181-6945 (imprimé)

1705-6500 (numérique)

Découvrir la revue

Citer cet article

Hoare, S. (1993). Romance du livre et du film : l'adaptation de la Romance du livre et de l'épée par Ann Hui. Cinémas, 3(2-3), 141-155.

https://doi.org/10.7202/1001196ar
Résumé de l'article

Cet article illustre le rapport entre cinéma et littérature dans l'oeuvre d'une des cinéastes « nouvelle vague » de Hongkong. Acclamée internationalement, la réalisatrice Ann Hui a adapté un roman populaire d'arts martiaux de Jin Yong, Romance du livre et de l'épée, en deux films : Romance du livre et de l'épée et Princesse Parfum (1987). Ne consistant pas simplement en la reproduction du roman originel, les deux films gagnent en signification par leur contraste par rapport à l'oeuvre de Jin Yong. 


\title{
Romance du livre et du film : l'adaptation de la Romance $d u$ livre et de l'épée par Ann Hui
}

\section{Stephanie Hoare}

\begin{abstract}
RÉSUMÉ
Cet article illustre le rapport entre cinéma et littérature dans l'œuvre d'une des cinéastes «nouvelle vague» de Hongkong. Acclamée internationalement, la réalisatrice Ann Hui a adapté un roman populaire d'arts martiaux de Jin Yong, Romance du livre et de l'épée, en deux films : Romance du livre et de l'épée et Princesse Parfum (1987). Ne consistant pas simplement en la reproduction du roman originel, les deux films gagnent en signification par leur contraste par rapport à l'œuvre de Jin Yong.
\end{abstract}

\begin{abstract}
This paper demonstrates that in the case of at least one Hongkong new wave director's work, the relationship between film and literature is of great importance. Internationally acclaimed Hongkong director Ann Hui has adapted a popular martial arts novel by Jin Yong, Romance of Book and Sword, into two films, Romance of Book and Sword and its sequel Princess Fragrance(1987). Hui does not simply try to duplicate Jin Yong's original narrative; in fact Romance and Princess Fragrance gain in meaning from their contrasting relationship to Jin Yong's novel.
\end{abstract}

La réalisation en deux parties par Ann Hui de la Romance du livre et de l'épée (Shujian en chou lu $)^{1}$, soit Romance du livre et de l'épée (Shujian en chou lu) et Princesse Parfum (Xiangxiang 
gongzhu), s'avère un exemple parfait, à la fois du rapport et de l'indépendance de l'adaptation d'un film en regard de l'œuvre littéraire. Ann Hui se sert du célèbre et populaire roman de Jin Yong pour créer ses propres œuvres personnelles et politiques par l'art du film ${ }^{2}$. Hui modifie certes le récit pour exprimer ses propres idées et plus encore, elle se sert du contraste productif entre film et roman pour fournir à un auditoire déjà renseigné sur l'histoire du roman un supplément de sens propre au langage cinématographique.

Comme plusieurs autres films de Hongkong des années 80, Romance du livre et de l'épée et Princesse Parfum reflètent les questions politiques de l'heure à Hongkong mais vont au-delà des craintes et des appréhensions de l'auditoire face à 1997 . En réponse à ma question sur les mobiles de cette adaptation, Ann Hui écrivait:

\begin{abstract}
Mon intention initiale et finale n'était pas vraiment d'essayer de faire une «adaptation-lecture» du roman.(...). [En fait, je voulais] utiliser les personnages et le cadre familier pour exprimer un thème fort différent, voire opposé à l'original. Tel que je le lis, le roman original est très proche des romances héroïques, mais moi, j'ai essayé de mettre ces personnages «héroïques» dans un contexte historique afin de les lire comme des figures tragiques délaissées par l'histoire et luttant en vain pour une cause perdue 3 .
\end{abstract}

On notera l'utilisation de guillemets pour le mot «héroïque». En effet, les personnages de Hui sont, comme tout être humain, bien moins héroïques et beaucoup plus complexes que ceux de Jin Yong, et les circonstances historiques auxquelles ils font face ne se présentent pas aussi simplement. Le lecteur du livre adapté à l'écran se rend inévitablement compte de ces différences, de ce contraste, inattendu et peut-être non souhaité, créateur de complexités politiques et humaines. Le visionnement d'un film peut susciter une certaine forme d'idéalisation. Or Ann Hui nous présente plutôt des situations prosaïquement humaines.

Les études sur l'adaptation de romans à l'écran ne trouvent généralement pas grâce auprès de spécialistes des questions cinématographiques. Car, par le passé, de telles études se sont souvent concentrées autour des questions de reproductions plus ou moins fidèles au modèle littéraire. Le cinéma et la littérature utilisent des techniques différentes dans leur communication du sens; les correspondances entre les techniques cinématographiques et les tropes littéraires atteignent vite les limites de ce projet quant à la visée d'une fidélité absolue (Andrew, p. 101). Dans Novel into Film, George Bluestone va jusqu'à suggérer que les cinéastes 
«abandonnent entièrement l'adaptation et écrivent directement pour l'écran» (p. 218) afin d'éviter complètement cette question.

Cependant, la popularité de l'adaptation littéraire au cinéma ne montre aucun signe de ralentissement et, dans le cinéma chinois, ce phénomène est trop répandu pour être négligé. Cette pratique influence la nature même du cinéma chinois dans son ensemble et atteint certains films individuels jusque dans la façon qu'ont les auditoires chinois de les visionner. Ailleurs, j'ai montré comment les cinéastes de Taiwan et de la République populaire de Chine ont utilisé l'adaptation littéraire pour cibler certains objectifs dans leurs films ${ }^{4}$. Les cinéastes de Hongkong, quant à eux, recourent moins souvent à l'adaptation, et les œuvres adaptées proviennent majoritairement de la littérature dite populaire ${ }^{5}$, contrairement aux cinéastes de Taiwan et de la République populaire, puisant dans la littérature dite «sérieuse». Cependant, la nouvelle vague de cinéastes hongkongais semble vouloir suivre ce courant amorcé par d'autres réalisateurs chinois. L'étude de ces adaptations nous révélera donc des similarités parmi les réalisateurs chinois de diverses origines.

Dudley Andrew lance un appel afin que les études de l'adaptation se renouvellent au-delà de la question de la reproduction fidèle, et amorcent une discussion plus productive sur le choix de ces cinéastes quant à la création d'un récit filmique et l'autonomie du film comme œuvre d'art (pp. 97-98).

Une définition de David Bordwell sur le récit filmique va me permettre de faire des liaisons plus fines entre le roman Romance du livre et de l'épée et les films de Ann Hui. Cette définition compare le récit d'un film à celui d'un roman sans faire de correspondances gauches et inexactes entre les techniques filmiques et les tropes littéraires. Le modèle de Bordwell, puisant aux théories constructivistes et formalistes russes, se compose de trois éléments : 1) l'ensemble événementiel d'une histoire que l'œuvre propose au spectateur (Bordwell le désigne par syuzhet); 2) le «style», la technique que l'œuvre utilise pour communiquer ces événements; et 3) l'ensemble événementiel de cette histoire telle que lue et reçue par le spectateur (fabula). Le style peut différer du livre au film, mais l'on pourra comparer avec succès les événements présentés par le récit filmique à ceux présentés par le roman (Bordwell, p. 51). J'utiliserai cette définition du récit comme base pour mes comparaisons entre les événements présentés dans les films de Hui et les événements dans le roman de Jin Yong afin de faire la lumière sur la singularité politique de la cinéaste. 
Ann Hui a réalisé Romance du livre et de l'épée et sa suite pour un auditoire déjà au fait de l'histoire, et ce d'une manière ou d'une autre. Cette connaissance supposée influe obligatoirement sur la signification des films. Plusieurs des spectateurs des films ont lu le roman de Jin Yong. Jin Yong est le nom de plume de Cha Liangyong, ou Louis Cha, le rédacteur du prestigieux journal Minghao; il est l'auteur des romans de cape et d'épée (wuxia) les plus appréciés de Chine. Même si Jin Yong vit et publie à Hongkong, et qu'il a arrêté d'écrire des romans de cape et d'épée dès les années 60 , ses œuvres romanesques continuent d'avoir un grand auditoire à Taiwan et en Chine continentale, tout comme à Hongkong. Son auditoire transcende les différents niveaux d'éducation, les classes sociales, les frontières politiques. Les romans de Jin Yong, remplis d'action, satisfont cet auditoire de littérature populaire; ils méritent aussi l'admiration des intellectuels pour leur érudition, leur connaissance de l'histoire et de la littérature classique, leur maîtrise du style. Un sondage informel parmi divers auteurs et cinéastes chinois «sérieux» de Taiwan et de République populaire de Chine révèle qu'ils ont quasiment tous lu les œuvres de Jin Yong et qu'ils les affectionnent, même les non-initiés à la littérature d'arts martiaux. L'auditoire de Ann Hui touche tout aussi bien les cinéphiles intellectuels que les fervents de littérature d'arts martiaux; plusieurs d'entre eux ont lu tous les ouvrages de Jin Yong, y compris Romance du livre et de l'épée.

Romance du livre et de l'épée est le premier roman de cape et d'épée de Jin Yong, qui fut publié en 1955; il continue à être un des romans les plus populaires du genre. L'intrigue, longue et compliquée du roman, comme celle de la majorité des romans de cape et d'épée, se veut pleine de personnages et de rebondissements inattendus. Je veux souligner ici certains aspects de l'intrigue, pertinents pour mon étude. Située durant le règne de l'empereur Qian Long (1736-1796), l'histoire raconte les exploits d'un groupe de 14 héros hors-la-loi. Ils sont les dirigeants d'une société clandestine han, nommée la Société fleur rouge. Leur mission consiste à renverser la cour impériale des Qing mandchous pour redonner le pouvoir aux Hans. Leur jeune chef, Chen Jialuo, est en fait le frère cadet inavoué de l'empereur Qian Long, un Han déguisé en Mandchou. Tout au long de ce roman de plus de 800 pages, Chen tente de convaincre son frère aîné d'être fidèle à son héritage han; aussi Chen et ses camarades doivent-ils affronter toutes sortes de difficultés, d'ennemis et de désastres naturels pour atteindre leur but. Malgré leur sagesse, leurs prouesses en arts martiaux, la justesse de leur cause, ils ne peuvent rivaliser avec le 
pouvoir de la cour des Qing et doivent donc se retirer à l'Ouest, vers un avenir incertain.

En plus de l'action indispensable aux arts martiaux, le roman raconte plusieurs trames amoureuses impliquant Chen et divers membres de la Société fleur rouge. La Société est aidée dans sa mission par un grand nombre d'alliés et de sympathisants, comprenant notamment le chef ouïgour Mu Zhaolun et ses deux filles Huo Qingtong et Kasili, également connue sous le nom de "Princesse Parfum". Huo est une guerrière, adepte des arts martiaux et de stratégie militaire. Chen et Huo s'intéresseront pudiquement l'un à l'autre une fois que Chen et ses camarades auront aidé les Ouïgours à recouvrer une copie sacrée du Coran. Au moment de leur séparation, elle lui offrira une précieuse épée de famille comme symbole de leur amitié, d'où le titre du roman. Plus tard, cependant, Chen croira à tort que Huo est engagée à un autre prétendant. Aussi, lorsqu'il rencontrera Kasili, qui est aussi belle que Huo estbrillante, se sentira-t-il libre de répondre à ses avances. Lorsque Huo et Chen redécouvriront tous deux la vérité de leur amour, il sera trop tard. Kasili aura déjà revendiqué Chen comme son fiancé, dans une cérémonie ouïgoure. Chen et Huo, à leur grand regret, acceptent ces circonstances pour le bien de Kasili. À la fin du roman, l'empereur Qian Long s'intéresse à Kasili et force Chen à renoncer à son droit sur elle, en échange d'une promesse de renverser les Mandchous. Kasili découvre que Qian Long n'a pas l'intention de garder sa promesse, et meurt dans une tentative, éventuellement couronnée de succès, pour en avertir Chen.

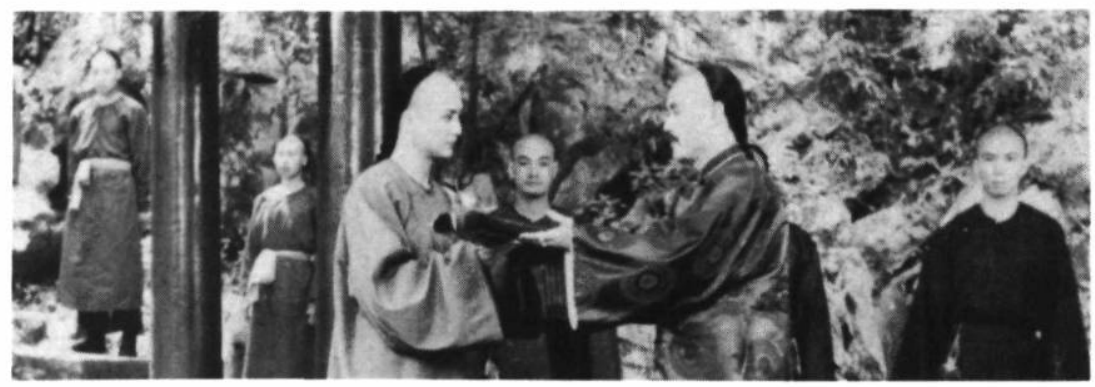

Romance du livre et de l'épée d'Ann Hui (1987) 
Cette histoire, par sa combinaison d'amours non partagées et de nobles causes perdues, s'est avérée populaire. La preuve de son succès a été ses fréquentes adaptations au petit et grand écran: une série télévisée hongkongaise de 1976, le film de Ann Hui de 1987, une autre série télévisée hongkongaise en 1987, et une série télévisée taiwanaise présentement en cours. Les auditoires de Hongkong se souviennent avec plaisir de la série télévisée hongkongaise de 19876 .

Ann Hui a tenu compte de la popularité à la fois du roman et des adaptations lorsqu'elle a fait sa version de Romance; elle-même avait lu le roman et elle avait vu la série de 1976. Elle savait jusqu'à un certain point que ces œuvres influenceraient la façon dont l'auditoire comprendrait son propre film ${ }^{7}$. La conscience de cette familiarité de l'auditoire avec les diverses formes de l'histoire peut être vue dans le traitement du récit lui-même par la cinéaste. Hui réduit la plupart des 14 membres importants de la Société fleur rouge à des rôles mineurs, certains même à des rôles muets. Un auditoire attentif, connaissant bien le roman ou les adaptations télévisuelles, peut identifier tous les 14; de plus, les 14 sont nommés individuellement dans le générique. Mais ceci n'est pas le seul clin d'œil aux passionnés de Jin Yong, ni le plus efficace, puisque même un observateur ignorant l'histoire peut parfaitement bien comprendre le film sans connaître l'identité exacte de chacun des 14 chefs de la Société fleur rouge. L'utilisation que Hui fait des flash-back démontre, de façon encore plus évidente, la conscience de cette connaissance, par l'auditoire, du contexte. Par exemple, dans une très courte scène sans dialogue, nous voyons Chen Jialuo, enfant, partant avec maître Yu Wanting (son prédécesseur comme chef de la Société), alors que sa mère, debout, le regarde. Comme elle le fait typiquement, Ann Hui ici n'indique pas que la scène est un flash-back par la cinématographie ni par le montage; la concision et l'absence de dialogues la rendent encore plus difficile à comprendre pour le non-initié. Un spectateur familier de l'un ou de l'autre des modèles du récit, du roman ou d'une série télévisée, peut immédiatement reconnaître le sens de l'événement: la famille de Chen était en danger parce que la cour mandchoue ne voulait pas que les vraies origines de l'empereur Qian Long soient connues du public. Aussi, sa mère avait-elle demandé au maître Yu d'éloigner Chen de son lieu natal et de l'élever comme un membre de la Société. Ann Hui reconnaît donc à la fois les antécédents du récit et leur connaissance par l'auditoire.

Cependant, les deux films de Hui font beaucoup plus que de simplement refléter les acquis de l'auditoire supposé : ils 
interagissent avec ceux-ci pour produire un autre sens. Hui modifie le récit pour exprimer sa propre vision de la réalité. L'auditoire dont l'attente est basée sur le roman original se voit déconcerté par les modifications du récit par Hui; ces modifications ressortent pour mettre en lumière le message de Ann Hui.

Comme beaucoup de films de Hongkong des années 80, Romance du livre et de l'épée et Princesse Parfum mettent en parallèle la situation historique actuelle. Hongkong est toujours pour le moment une colonie britannique, mais en 1997 elle va retourner à la Chine, son appartenance d'origine. Ce changement mettra le Hongkong capitaliste sous le contrôle du gouvernement communiste de la République populaire de Chine. Quoique le gouvernement chinois actuel ait accepté de ne pas restructurer Hongkong pour une période de 50 ans après 1997, le gouvernement de la république populaire de Chine est reconnu pour ses changements abrupts de politique lors d'une passation de pouvoir. Li Cheuk-to et d'autres critiques ont démontré comment les films de Hongkong peuvent refléter le statut colonial de Hongkong et les angoisses des auditoires hongkongais quant à leur avenir8; Li a lui-même constaté les connotations politiques de Romance de Ann Hui ${ }^{9}$. Quelques-unes des modifications majeures de Ann Hui au récit, situé au XVIIIe siècle et rédigé en 1955 par Jin Yong, mettent en effet l'accent sur cette pertinence du récit pour le Hongkong contemporain.

Hui a dû réduire la trame de l'intrigue à cause de contraintes temporelles propres au long métrage, mais les parties du récit sur lesquelles elle se concentre fournissent des parallèles évidents avec des questions politiques actuelles. Le roman original est en grande partie l'histoire de la Société fleur rouge et de ses alliés; ceux-ci sont tous des individus différenciés et le roman s'attarde à raconter en détail les activités de plusieurs d'entre eux. Hui se concentre sur les relations de Chen Jialuo avec l'empereur Qian Long et avec les Ouïgours. Chen est un Chinois han de la Chine septentrionale. Depuis son enfance, sa mission a été de renverser le gouvernement étranger Qing et de restaurer le gouvernement chinois han. Sa motivation trouve sa force dans la haine et la tyrannie, et dans la conviction que ce sont les Chinois hans qui doivent gouverner la Chine. Chen, témoin de l'agression impérialiste des Mandchous à l'égard des Ouïgours, tourne naturellement sa sympathie vers ces derniers; les rebelles hans et les tribus occidentales s'unissent dans leur combat contre le gouvernement étranger. Cette intrigue, loin d'être un modèle précis pour la politique contemporaine, éclaire certaines questions contemporaines : un pouvoir étranger impose 
son gouvernement à des cultures différentes de la sienne, comme les Britanniques étrangers ont imposé leur pouvoir et leur culture à Hongkong. La sympathie des gens en faveur d'un gouvernement autonome va naturellement vers d'autres victimes de colonisation.

Chen, découvrant que Qian Long est son frère, croit avoir trouvé le moyen d'accéder au gouvernement han autonome. L'empereur mandchou se révèle être, en fait, un empereur chinois han déguisé, et tout ce qu'il reste à faire, c'est de le convaincre d'agir conformément à son rôle. Mais Chen ne réussit jamais à changer Qian Long, qui demeure culturellement et politiquement un Mandchou, qui gouverne comme ses prédécesseurs l'ont fait. Un tyran demeure tyrannique, peu importe son origine ethnique. La République populaire de Chine, malgré le fait qu'elle soit chinoise, demeure une puissance étrangère imposant sa volonté sur d'autres cultures sans pouvoir.

Ann Hui met davantage de l'avant la dimension politique de son récit, en y ajoutant des dialogues ouvertement politiques. Dans une scène de Romance, un ajout au récit d'origine, Hui commente l'apathie politique de la plupart des Chinois de Hongkong. Chen et Huo Qingtong marchent incognito dans les rues de Hangzhou, en regardant la foule vaquer à ses occupations quotidiennes. Huo note leur allure satisfaite et Chen se lamente ainsi : «Ils ont déjà oublié les torts infligés à leur pays qui n'ont pas encore été vengés.» Il est intéressant de noter que le sous-titre anglais pour cette phrase se lit ainsi : "Yes, as long as they have enough to eat.» Quoique complètement différentes, les phrases chinoises et anglaises déplorent la suffisance débilitante d'un peuple colonial.

La dernière phrase de Princesse Parfum, un autre ajout au récit, énonce clairement sa position politique. En expliquant à l'empereur pourquoi il l'a épargné, Chen Jialuo déclare avec dégoût : "N'eût été de toi, on aurait pu avoir un empereur pis encore.» Ceci fait écho au cynisme de la colonie face aux gouvernants étrangers.

Quoique plusieurs des ajouts de Hui au récit commentent et renforcent les parallèles à des questions d'aujourd'hui, sa Romance du livre et de l'épée est plus qu'une diatribe politique. Cette adaptation se veut une déclaration plus universelle, plus humaniste: les êtres humains et leurs rapports, et ce en tout temps, ne sont pas aussi parfaits qu'on pourrait s'y attendre ou qu'on aimerait le croire. Les camps politiques ne se définissent pas aussi clairement, les situations humaines sont toujours compliquées et ne trouvent pas toujours de solutions adéquates. Pour comprendre plus clairement ce message, il suffit de comparer le film et le roman. 
Dans le roman, les camps sont assez bien définis, spécialement à la fin : la Société fleur rouge, du côté de la justice et du peuple han, combat la cour mandchoue corrompue. De même, les individus dans chaque camp sont sans équivoque. Chen Jialuo, le chevalier errant, héros exemplaire, incarne toutes les vertus dictées par le code de l'honneur. Il est brave dans les situations mettant sa vie en péril, et se bat contre plusieurs illustres guerriers à travers le roman. Il est loyal, toujours fidèle à la Société fleur rouge, même s'il ressent une affection fraternelle pour Qian Long. Il est chaste et se conduit en gentilhomme avec les femmes, plus spécifiquement avec les sœurs ouïgoures. En outre, il est un lettré cultivé, versé dans les classiques confucéens, un administrateur compétent pour la Société et un négociateur talentueux dans ses tractations tant avec ses alliés qu'avec ses ennemis.

Le Chen de Ann Hui possède les mêmes prouesses en arts martiaux, des manières raffinées et une bonne nature. Une description physique du Chen du roman convient aussi bien au Chen de Hui:

L'allure de ce jeune chef était tout simplement celle d'un jeune homme bien élevé de famille riche et son langage était, en plus, fort recherché; il n'était pas du tout de la même étoffe que ces bandits rustiques [de la Société fleur rouge] (p. 84).

Comme le Chen de Jin Yong, celui de Hui est vêtu plus proprement et plus élégamment; il est plus poli et posé que les autres bandits.

Dans leur comportement éthique, cependant, les deux Chen sont grandement différents. Le Chen du roman de Jin Yong suit de façon significative le code d'honneur du chevalier errant, le Chen du film fait face à des situations qui rendent cette conduite difficile à suivre. Hui change les circonstances entourant les relations de Chen avec les sœurs ouïgoures, de façon à humaniser son personnage. Dans le roman, Chen est attiré par Huo Qingtong, mais il croit qu'elle s'intéresse à un autre jeune homme; ce dernier est en réalité une jeune femme déguisée, une simple amie de Huo; mais Chen ne l'apprend que lorsqu'il est déjà lié à la sœur de Huo, Kasili. Le Chen de Jin Yong est lui aussi partagé dans ses amours avec les sœurs, mais au moins le triangle amoureux est le résultat d'une coïncidence malheureuse; on peut présumer qu'il aurait été fidèle à Huo s'il n'y avait eu cette circonstance exceptionnelle. Les films de Hui, par contre, omettent complètement le personnage de la jeune femme déguisée. Dans Romance de Hui, on voit par son attitude et ses agissements que Huo s'intéresse à Chen: elle le regarde avec langueur lorsqu'il ne regarde pas; comme dans le roman, elle lui fait cadeau d'une épée précieuse. La réaction de Chen, toutefois, semble poliment neutre. Lorsqu'il s'engage avec 
Kasili, nous pouvons donc en déduire que son agissement est une question de préférence personnelle, le résultat non tant de circonstances extérieures que de sa propre nature. Il peut être critiqué pour son choix là où sa contrepartie romanesque ne le peut.

De même que le Chen de Jin Yong est au-dessus de tout reproche, son empereur Qian Long est clairement un méchant. Qian Long n'expose jamais son idéologie personnelle dans le roman, mais une grande partie peut être déduite à partir de ses pensées et actions. Son intérêt principal dans le gouvernement n'est pas le bien-être du peuple, l'idéal confucéen, mais simplement la gloire personnelle. Il désire être l'émule des grands empereurs Wu des Han et Taizong des Tang, seulement parce qu'ils étaient reconnus comme de grands lettrés, guerriers et conquérants (pp. 283, 438-439); il ne respecte pas leur croyance en des valeurs confucéennes, ni leur conduite envers le peuple. Il est habitué à la révérence servile de tout un chacun de sa cour, à l'exception de l'impératrice douairière, et il ne peut accepter de critiques d'aucune sorte.

L'interaction entre les deux personnages dans le roman illustre le contraste frappant entre leurs valeurs. Dans ses conversations avec son frère, Qian Long ne révèle aucune préoccupation pour le peuple, mais seulement un intérêt pour son avancement personnel. Cette attitude donne à Chen l'occasion de jouer le rôle de défenseur du peuple, un rôle dans lequel il a habituellement le dernier mot. Lors de l'une de leurs premières rencontres, Qian Long exprime de l'admiration pour l'empereur Taizong des Tang, en raison de ses habiletés dans les lettres, de ses prouesses militaires et de ses succès dans la construction de l'empire chinois. Qian Long espère secrètement passer à l'histoire comme un autre Taizong. Chen Jialuo lui répond par un avertissement voilé:

J'ai lu les Politiques importantes du règne Zhenguan, la biographie de Taizong, et j'ai trouvé qu'il y avait plusieurs phrases fort sensées. Qian Long, réjoui, lui demanda : "Quelles phrases?» (...) Chen Jialuo dit : «L'empereur Taizong a dit : "Un bateau est comme le souverain du peuple, et l'eau comme les masses : l'eau peut porter le bateau, et peut aussi le faire chavirer." Il a également dit : "Si le Fils du ciel a la Voie, le peuple le choisira comme son dirigeant; s'il ne l'a pas, ils vont s'en débarrasser. Ceci doit sincèrement être redouté."» Qian Long fut silencieux (pp. 283-284).

Par cette citation érudite, Chen condamne indirectement les ambitions de Qian Long et exprime ses propres préoccupations politiques moralement justes. Qian Long, qui n'est pas habitué à la critique, ne peut rien dire en sa défense. 
Le contraste créé par Hui entre Chen et Qian Long n'est pas si clair. Même si le Chen de Jin Yong promet au début de voir à ce que rien n'arrive à Qian Long, sa première loyauté va toujours à la Société fleur rouge, et sa relation avec son frère n'occasionne jamais de disputes avec ses camarades hors-la-loi. Hui ajoute de nouvelles dimensions aux valeurs de Chen: dans Romance de Hui, Chen a initialement plus confiance en Qian Long que sa contrepartie romanesque. Il l'appelle d'abord «dage», grand frère, dès qu'il apprend leur véritable lien; le Chen de Jin Yong ne le fait que lorsqu'il tente de convaincre Qian Long de se joindre à la cause de la Société.

Le Chen de Hui essaie aussi de convaincre son frère de renverser le gouvernement Qing, et ce même plus tôt que le Chen de Jin Yong. Mais il obtient de Qian Long une réaction autrement différente qui influe de façon décisive sur ses agissements ultérieurs. Dans Romance de Hui, lorsque Chen et Qian Long découvrent qu'ils sont frères, Chen lui demande de l'aider à renverser les Mandchous. Qian Long répond en donnant sa version de la situation du moment :

\begin{abstract}
QIAN LONG. - Il n'y a aucun conflit entre le fait d'être han et d'être empereur d'une cour mandchoue. La prospérité du pays en témoigne.

CHEN JIALUO. - Mais maintenant les Mandchous massacrent les Chinois han.

QIAN LONG. - Jialuo, je te demande : les Ming, c'était une dynastie chinoise - pourquoi s'est-elle effondrée? De plus, on peut pardonner l'effondrement d'une dynastie; mais en détruire une qui est paisible et prospère, quelle raison y aurait-il? Nos cartes territoriales sont maintenant conformes à celles de la dynastie Tang à son apogée. Pendant mon règne, il n'y a eu aucune augmentation d'impôts; c'est connu de tous. Y a-t-il quelqu'un qui ne veuille porter la queue mandchoue ou raser son front? Ou encore quelqu'un qui refuse de passer les examens et de devenir officiel du gouvernement? Et puis, si nous nous rebellons, nous ne pouvons être sûrs de la victoire. Comment pourrais-je me décider à détruire un pays paisible et prospère juste parce que je suis un Chinois han?
\end{abstract}

Dans la scène équivalente du roman, Qian Long n'essaie jamais de justifier ses agissements; il essaie tout simplement d'éloigner Chen de la Société en lui proposant tendancieusement un poste de haut officiel. Cette offre lui semble être le meilleur argument qui puisse être. Le Qian Long de Ann Hui, en revanche, présente un ensemble de valeurs politiques que Chen et l'auditoire peuvent soupeser. Ces vues sont conformes à celles d'un empereur humaniste suivant les idéaux de Confucius et de Mencius: le bien- 
être et l'assistance au peuple sont la priorité de l'empereur et Qian Long démontre qu'il l'a réalisée.

Après avoir soupesé l'argument de son frère, le Chen de Ann Hui y consent et crée donc un conflit politique entre lui-même et les autres membres de la Société fleur rouge. Hui clarifie ce conflit également par l'ajout de dialogues. Lorsque Wen Tailai, quatrième en rang dans la Société, accuse Chen de loyauté excessive à Qian Long, la discussion suivante a lieu :

CHEN.- Pourquoi voulons-nous renverser les Qing? Juste pour pouvoir reporter des vêtements hans? Si la rébellion n'amène que souffrance au peuple, je préfère ne pas me rebeller.

WEN. - Tu as été trop influencé par ton frère. As-tu oublié ce que les Mandchous nous ont fait, à nous, Hans? À Yangzhou, ils ont tué 800000 d'entre nous. Dans les trois massacres Jiading, 200000 en dix jours. Ceux qui n'ont pas rasé leurs têtes les ont perdues. Est-ce que les Mandchous pourraient me regarder en face?

CHEN. - Quel fondateur d'une dynastie chinoise han n'a pas tué des gens? Regarde les empereurs Ming avec leurs armées et leur police secrète. Eux aussi ont massacré de bons officiels qui défendaient le peuple. Ça n'a pas d'importance qui est empereur, à condition qu'il soit un bon empereur.

Cette scène du film montre une rupture idéologique entre Chen et la Société, qui n'existe pas dans le roman. Wen Tailai exprime ici la motivation qui sous-tend les buts de la Société fleur rouge. Sa plus grande préoccupation consiste à venger les injustices commises contre sa race, alors que Chen met l'idéal de Mencius au-dessus de l'ethnicité et des torts du passé.

Le Chen de Hui accorde grande foi aux arguments humanistes de Qian Long sur le gouvernement alors que le Chen de Jin Yong ne vacille nullement devant les offres de hauts postes officiels de Qian Long. La relation familiale et le bien-être du peuple sont plus importants pour le Chen de Hui que le nationalisme han et la vengeance, et suffisamment importants pour qu'il questionne le jugement de ses confrères hors-la-loi, avec lesquels il devrait être en parfait accord d'après le code chevaleresque. Hui nous présente donc un héros divisé.

Le Chen de Hui perd vite la confiance initiale qu'il avait mise en Qian Long. Et avec la Société fleur rouge, il finit par extorquer à Qian Long une promesse de se plier, mais ses valeurs conflictuelles demeurent et influencent la progression du récit jusqu'à la toute fin. La confiance qu'a la Société, encouragée par Chen, que Qian Long tiendra sa parole, mène en fait à sa destruction. Â la fin de Princesse Parfum, la Société accepte l'invitation de Qian Long à son palais, et ses membres sont fusillés par les soldats du palais. Le Chen de Jin Yong ne fait pas aussi confiance à Qian Long. Après 
avoir appris la mort de Kasili, Chen jure de se venger en tuant l'empereur. Il ne réussit pas à le faire, mais il réussit à faire sortir indemne de Beijing la majorité de la Société fleur rouge. Le Chen de Hui, par contre, même après la dernière manifestation de trahison de Qian Long, va à l'encontre du code chevaleresque de loyauté et de vengeance en l'épargnant. Il le fait en vertu de ses valeurs humanistes : sa dernière phrase indique non pas qu'il craint pour lui-même, mais qu'il craint pour le peuple les conséquences d'un remplacement de Qian Long.

Il faudrait noter qu'Ann Hui n'a pas modifié de façon significative les valeurs politiques de Chen, mais seulement les circonstances dans lesquelles elles entrent en jeu. Dans le roman, Qian Long n'invoque jamais l'humanisme de Chen, aussi Chen n'a jamais à décider entre Qian et la Société. Ce sont les modifications des circonstances autour de Chen, plus réalistes et complexes, qui rendent difficiles son adhésion totale à l'idéal du chevalier errant.

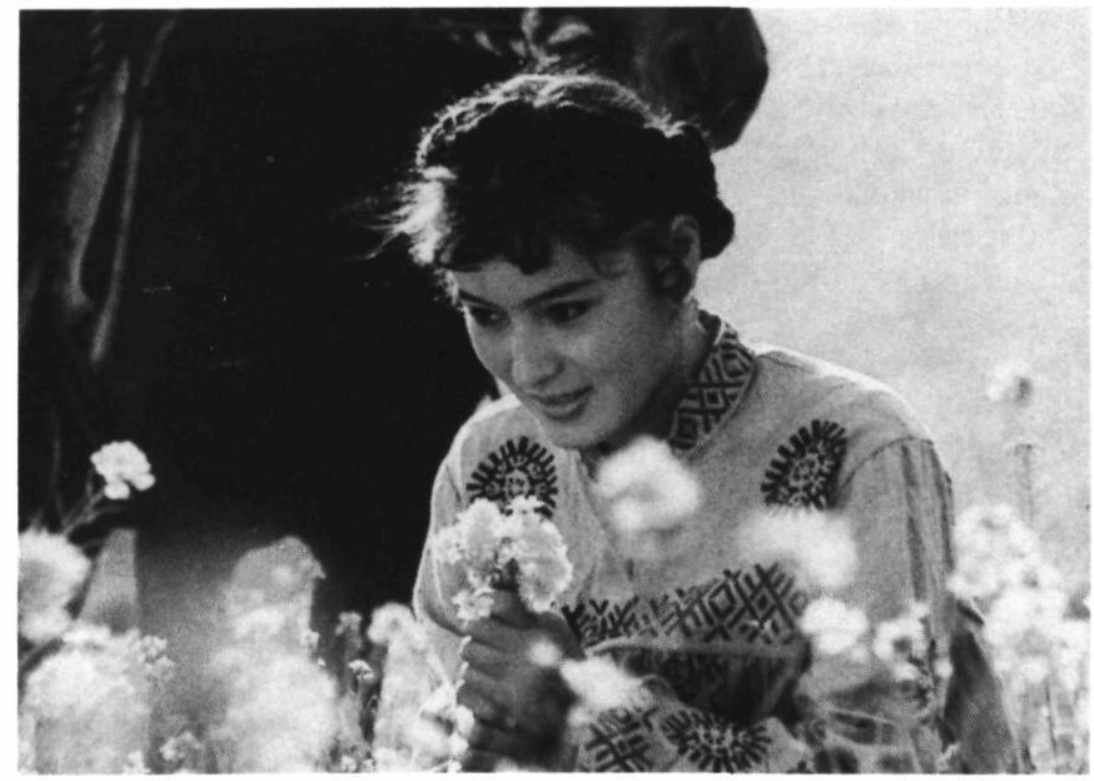

Princesse Parfum d'Ann Hui (1987)

En modifiant les circonstances de Chen, Ann Hui a aussi modifié le personnage de Qian Long. L'empereur Qing de Hui est moins régi par sa soif du pouvoir. Tel qu'on le voit, dans son langage 
tout au moins, il est préoccupé par la qualité morale de son gouvernement. Son attitude envers son frère fournit un contraste supplémentaire au roman. Dans la version de Jin Yong, le projet final de Qian Long est de liquider la Société fleur rouge, y compris Chen Jialuo. Qian Long est prêt à tuer son propre frère pour conserver le pouvoir, et on peut le voir dans toute sa noirceur. Le Qian Long du film d'Ann Hui, par contre, est plus ambigu. Bien qu'il soit déterminé à liquider la Société fleur rouge, Qian Long ne peut se décider à tuer son frère. Dans la scène finale de Princesse Parfum, Qian Long fait tuer par ses troupes tous les membres de la Société, sauf Chen. Même si, ce faisant, il donne l'occasion à Chen de le tuer. Le Qian Long de Hui, comme le Chen Jialuo de Hui, détiennent des valeurs plus conflictuelles que leurs prédécesseurs romanesques : les liens familiaux et l'affection personnelle triomphent sur la politique et le maintien du pouvoir.

Qian Long n'est pas qu'un méchant avare; il est un être humain capable de certaines actions morales. Et Chen Jialuo n'est pas le héros purement chevaleresque du roman de Jin Yong, mais un jeune homme moins conséquent, quoique bien intentionné, qui fait face à des choix difficiles. Le contraste qu'offrent les films d'Ann Hui par rapport au roman original donne clairement à voir aux spectateurs renseignés que la vie, à n'importe quel moment de l'histoire, n'est pas aussi simple et parfaite que nos idéaux. L'adaptation d'Ann Hui de Romance du livre et de l'épée n'est pas une simple réplique du roman original; elle exprime la position et l'art d'Ann Hui. Pourtant, elle s'enrichit sémantiquement par la comparaison, par son rapport, donc, avec le roman de Jin Yong. Romance du livre et de l'épée et Princesse Parfum d'Ann Hui sont des œuvres d'art en soi, mais leurs liens avec la littérature ne sauraient être ignorés.

Colgate University, Hamilton, N. Y.

Traduit de l'anglais par Marie Claire Huot

\section{NOTES}

1 Jin Yong (Cha Liangyong), Shu jian en chou lu (Romance du livre et de l'épée)(Hongkong : Wuxia chubanshe, s.d.).

2 Romance du livre et de l'épée est un roman en deux volumes. Romance du livre et de l'épée de Hui couvre la majeure partie du premier volume et Princesse Parfum couvre le reste du récit original.

3 Ann Hui, lettre à l'auteure, 29 juin 1992. 
4 Stephanie Hoare, Melodrama and Innovation : Literary Adaptation in Contemporary Chinese Film. Thèse non publiée. Cornell University, 1989.

5 Une exception notable est un autre film d'Ann Hui, L'Amour dans une ville prise (Qing cheng zhi lian, 1984), adapté d'une nouvelle d'Eileen Chang.

6 Je fonde cette affirmation, principalement, sur des expériences personnelles. Plusieurs de mes amis de Hongkong ont louangé cette série en particulier; lorsque moi-même j'ai vu le film de Hui dans un cinéma de Hongkong en 1987, l'auditoire était visiblement excité de constater la présence de la vedette de cette série dans la salle. J'ai également visionné une courte rétrospective nostalgique de cette série, à la télévision de Hongkong, en janvier 1992.

7 Ann Hui, lettre à l'auteure, 29 juin 1992.

8 Esther Yau a également fait beaucoup de recherches sur ce sujet; son travail et celui de $\mathrm{Li}$ feront partie d'une collection à paraître aux presses universitaires de Cambridge, sous le titre provisoire Cinema and Social Change in Mainland China, Hongkong, and Taiwan. Voir aussi Li Cheuk-to, éd., The China Factor in Hongkong Cinema, Catalogue du $14 \mathrm{e}$ festival international de Hongkong (Hongkong, 1990), pour d'autres articles sur ce sujet.

9 Li Cheuk-to, «The Return of the Father : Hongkong Cinema and its Chinese Context in the 1980 s", communication non publiée présentée à la UCLA Conference on Chinese Cinema, 4-6 janvier 1990, p. 17.

\section{OUVRAGES CITÉS}

Andrew, Dudley. Concepts in Film Theory. Oxford : Oxford University Press, 1984.

Bluestone, George. Novel into Film. Baltimore : 1957.

Bordwell, David. Narration in the Fiction Film. Madison : University of Wisconsin Press, 1985.

Hoare, Stephanie. «Melodrama and Innovation : Literary Adaptation in Contemporary Chinese Film». Thèse non publiée. Cornell University, 1989.

Jin Yong (Cha Liangyong). Shu jian en chou lu (Romance du livre et de l'épée), 2 vol. Hongkong : Wuxia chubanshe, s.d.

\section{GLOSSAIRE DES PRINCIPAUX TERMES CHINOIS (par ordre alphabétique)}

Cha Liangyong

da ge

Jin Yong

Shujian en chou lu

wuxia

Xiangxiang gongzhu

Xu Anhua
查良庸

大哥

金庸

書㓣恩仇錄

武俠

香香公主

許鞍華 